\title{
Cancer therapy with chimeric antigen receptors - A landmark moment for cancer immunotherapy
}

\begin{abstract}
Immunotherapy has proven successful in inducing long-term remissions of hard-totreat cancers. The early identified protein receptor on the surface of T-cells (cytotoxic T-lymphocyte antigen 4, CTL-4) and a molecule (programmed death 1, PD-1) led to astonishing tumor shrinkage and increased survival, particularly in metastatic melanoma. Thus, anti CTL-4 and anti PD-1 have opened up new vistas in tumor treatment. Beyond that, genetically modified patients' T-cells and PD-1 molecules promise to be even more effective in specifically tailoring the treatment to the patient along the precepts of personalized medicine. CAR-therapy is now the subject of numerous clinical trials in the hope that, like the antibodies, it can target an assortment of cancers. This article essentially addresses the promises, implications and future prospects of the momentous introduction for the first time of gene therapy in the United States. However, notwithstanding the successes achieved so far, CAR therapy may not benefit everyone. It needs to be extended to a larger population of patients, applied to treat a wide range of diseases including HIV, certain infections, immune deficiencies and autoimmune disorders, cancers, including solid tumors, and rendered more affordable.
\end{abstract}

Keywords: immunotherapy, synthetic immunotherapy, acute lymphoblastic lymphoma, chronic lymphocyte leukemia, tumor infiltrating lymphocyte, cytokine release syndrome, cytokine storm, human gene therapy, human cell therapy, t-cell receptor, b-cell receptor, chimeric antigen receptor
Volume 8 Issue 6 - 2017

\author{
Alain L Fymat \\ International Institute of Medicine and Science, USA
}

Correspondence: Alain L Fymat, International Institute of Medicine and Science, California, USA, Tel (760) 485-9I49, Email alain.fymat@fiimas.org

Received: October 16,2017| Published: November 28, 2017
Abbreviations: ALL: acute lymphoblastic lymphoma; BLA, biologics license application; CAR, chimeric antigen receptor; CD19, specific antigen; CBER, center for biologics evaluation and research; CHOP, children's hospital of philadelphia; CLL, chronic lymphocytic leukemia; CRS, cytokine release syndrome; CTLA, cytotoxic T-lymphocyte antigen; DNA, deoxyribonucleic acid; ETASU, elements to assure safe use; FDA, (U.S.) food \& drug administration; FDCA, food, drug and cosmetic act; HGT, human gene therapy; HCT, human cell therapy; INDA, investigational new drug application; MHC, major histocompatibility complex; NCI, (U.S.) National Cancer Institute; PD, programmed death; REMS, risk evaluation and mitigation strategy; OCE, oncology center for excellence; PHS, (U.S.) public health service; PSHA, Public Health Service Act; REMA, risk evaluation and mitigation strategy; T-cell, a type of white blood cell (lymphocyte); TCR, T-Cell receptor; TIL, tumor infiltrating lymphocyte

Diseases Cited: Cancer, Acute lymphoblastic lymphoma, Chronic lymphocytic leukemia, Cytokine release syndrome

Drugs Listed: Actemra (tocilizumab), Kymriah (tisagenlecleucel)

\section{Introduction}

A recent article $^{1}$ described the newly emerging anti-cancer strategy of "immunotherapy" and its success in inducing long-term remissions of hard-to-treat cancers - currently in about one-third of patients. It also succinctly reviewed the history of immunotherapy from its early beginnings in 1987 with the identification of a new protein receptor on the surface of T-cells (a blocker), called cytotoxic T-lymphocyte antigen 4 (CTLA-4). In a twist on the technique, consisting of manipulating immunosuppression rather than strictly focusing on immunosuppression (that is, blocking the blocker), tumors in mice were erased by antibodies against CTLA-4. Separately, the discovery of the molecule programmed death-1 (PD-1), another blocker of
T-cells, led to astonishing tumor shrinkage and increased survival, including more particularly in cases of metastatic melanoma. AntiCTL-4 and anti-PD-1 have thus opened-up new vistas in tumor treatment.

However, with increased interest on genomic and personalized medicine, the question arose as to whether a personalized treatment involving genetically modified patient's T-cells could make them target tumor cells. The idea had its beginning in the pioneering work of Rosenberg (2010) at the U.S. National Cancer Institute (NCI), dubbed chimeric antigen receptor (CAR-T)-therapy, which has now become an important treatment avenue. CAR-therapy is now the subject of numerous clinical trials in the hope that, like the antibodies, it can target an assortment of cancers.

Now, more than six months after publication of, ${ }^{1}$ in one of its News Releases, ${ }^{2}$ the U.S. Food \& Drug Administration (FDA) announced its approval of the CAR-T cell therapy to treat certain children and younger adults with B-cell acute lymphoblastic leukemia (ALL). This announcement has heralded the advent of the first gene therapy in the U.S. The aim of this paper is to discuss the action taken by the FDA and elucidate its far reaching implications in treatments of cancer, and other serious and life-threatening diseases. To better appreciate this pioneering development, we will first provide a brief primer on childhood ALL, describe the CAR T-cell therapeutic approach, and discuss in greater lengths the FDA's historic action that made available in the U.S. the first gene therapy.

\section{Childhood acute lymphoblastic leukemia treatment}

This section provides a succinct primer on childhood acute lymphoblastic leukemia (ALL), a cancer of the bone marrow and blood, in which the body makes too many abnormal lymphocytes. NCI estimates that approximately 3,100 patients aged 20 and younger 
are diagnosed with ALL each year. ALL can be of either of T- or B-cell origin, with B-cell being the most common.

\section{Definition}

Childhood acute lymphoblastic leukemia (ALL) is the most common type of cancer in children in the United States. Leukemia may affect red blood cells, white blood cells, and platelets. Intensive chemotherapy cures more than $80 \%$ of children where ALL arose in B-cells, which is the predominant type of pediatric ALL. But, there are few treatment options for patients whose cancers do not respond to treatment or go into remission and later relapse. Also, past treatment for cancer and certain genetic conditions affect the risk of having childhood ALL.

\section{Characteristics}

In a healthy child, the bone marrow makes blood stem cells (immature cells) that become mature blood cells over time. A blood stem cell may become either a myeloid stem cell or a lymphoid stem cell. In turn, a myeloid stem cell becomes one of three types of mature blood cells: (a) red blood cells that carry oxygen and other substances to all tissues of the body, (b) white blood cells that fight infection and disease, and (c) platelets that form blood clots to stop bleeding. On the other hand, a lymphoid stem cell becomes a lymphoid blast cell and subsequently one of three types of lymphocytes: (a) B-lymphocytes that make antibodies to help fight infection, (b) T- lymphocytes that help B-lymphocytes make the antibodies, and (c) natural killer cells (K-cells) that attack cancer cells and viruses.

\section{Symptoms}

Signs of childhood ALL include fever and bruising.

\section{Diagnosis and prognosis}

Tests that examine the blood and bone marrow are used to detect and diagnose childhood ALL. Certain factors affect prognosis and treatment options. In a child with ALL, too many stem cells become lymphoblasts, B-lymphocytes, or T-lymphocytes. The cells do not work like normal lymphocytes and are not able to fight infection very well. These cells are cancer (leukemia) cells. Also, as the number of leukemia cells increases in the blood and bone marrow, there is less room for healthy white blood cells, red blood cells, and platelets. This may lead to infection, anemia, and easy bleeding.

Past treatment(s) for cancer and certain genetic conditions affect the risk of having childhood ALL. Anything that increases the risk of getting a disease is called a "risk factor". However, having a risk factor does not mean getting cancer and not having a risk factor doesn't mean not getting cancer.

\section{The chimeric antigen receptor approach}

This technology merges gene therapy, synthetic biology and cell biology in the laboratory.

\section{Materials and methods}

The CAR-T technology is a personalized therapy that involves the following four steps : (a) First, a batch of certain T-cells known to respond best to a given disease are extracted from the blood, (b) a custom-built virus is used to implant them with new genes, ${ }^{3}$ (c) cells are created that target a molecule (CD19) that is found on the surfaces of some cancers; and (d) finally, after patients undergo a "lymphodepleting" chemotherapy, they receive the infusion of the engineered immune cells where their new DNA gives them a fresh set of targets to attack.

\section{Approach}

If the treatment works as intended, the CAR T-cells will continue to multiply, increasing the therapy's potential for eradicating the cancer. CAR T has been tested in dozens of studies $(\sim 1,000$ patients) in certain types of cancers (leukemia and lymphoma). Half or more of these patients are now living longer than expected and hundreds appear to be cancer-free. ${ }^{4}$

In step (b) above of the CAR T approach, several custom-built viruses could be theoretically employed. Thus, based on the wellknown proclivity of HIV for infecting cells, Posey et al. ${ }^{5}$ have used the HIV virus.

\section{Results and discussion}

The end result here is made up of two parts: (a) An antibody-like part of CAR that juts out of the cell surface to bind to the cancer antigen of choice, and (b) the rest of CAR that plunges into and through the T-cell membrane to generate the proper signals, and activate the T-cells as soon as the cancer antigen is detected. The approach was successfully employed in a limited clinical trial against the surface protein CD19 (this protein is found on healthy B-cells as well as the malignant cells they might become). This is further evidence substantiating the theory that cancer is braided within our genome. It was tested in cases of chronic lymphocytic leukemia (CLL) and an expanded repertoire of cancers (Figure 1).

Many research groups have reported excellent results in advanced cases of leukemia and lymphoma (Fred Hutchinson Cancer Research Center, Memorial Sloan Kettering Cancer Research Center, National Cancer Research Institute, Seattle Children's Hospital, and University of Pennsylvania). The response rates vary by disease, age and medical condition of the patients, and the technique used. Thus, at the University of Pennsylvania, marked clinical improvement was noted in CLL cases and a $100 \%$ response rate after only one month in $90 \%$ of the children treated for ALL. Nonetheless, some relapses do occur and some ALL patients have died from treatment-related problems. The results of the CAR-T therapy, particularly in the case of ALL, have been summarized in. ${ }^{1}$

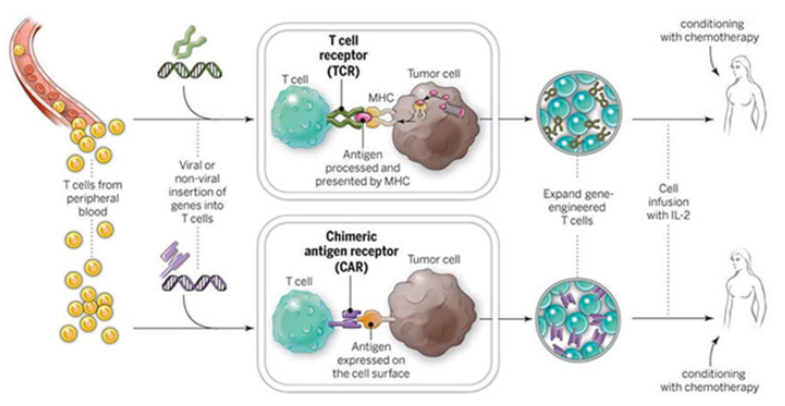

Figure I CAR T-cells and TCR T-cells are engineered to produce special receptors on their surfaces. They are then expanded in the laboratory and returned to the patient. (Source: National Cancer Institute).

\section{Conclusion}

CAR T-cells may have the potential to treat different types of diseases and cancers, including solid tumors, certain infections, and autoimmune diseases. However, the potential use of these treatments to treat hematologic malignancies, adult forms of leukemia and 
certain types of non-Hodgkin's lymphoma are not yet approved and are currently investigational.

\section{Limitations, refinements and improvements on the car-t technology}

\section{Limitations}

Although the ALL results have been impressive, the technology cannot readily be expanded to other cancers because for a T-cell to be primed to attack, it needs to be given precise co-ordinates. Otherwise, it may lock onto and destroy something else in the body. Besides CD19, which is found in only a few cancers, we currently know of no other chemical target that is specific to cancer alone. Another limitation consists in the associated toxicities of the treatment, primarily the toxic side effects of colitis and pneumonitis.

\section{Refinements}

To overcome the limitation due to the uniqueness of CD19, Kole Roybal (Cell, 2016) and his colleagues at the University of California, San Francisco are tweaking cells to attack but only when they sense two different target chemicals instead of one. In isolation, neither target may be unique to cancer cells - but the combination might be, which could allow the immune system to be unleashed on tumors whilst sparing healthy tissue. Another refinement of the technique would consist in overcoming the toxic effects that the treatments can trigger. Nonetheless, a concerning aspect is the known fact that as the number of T-cells doubles, roughly every 12 hours, a runaway immune reaction called a cytokine storm is triggered, which can be fatal to certain patients. The biggest cytokine storms seem to come from the patients with the most advanced cancers. The solution is then to give the sickest patients the lowest dose so that the T-cells multiply more slowly, reducing the chances of an immune-system overreaction. The CAR technology needs also to be extended to other types of cancer than heretofore examined, including breast cancer and the still unforgiving pancreatic cancer.

\section{Improvements}

While it has been a long way from the laboratory to the clinic, engineered T-cells might be used to treat a wide range of diseases, including HIV, immune deficiencies, and autoimmune disorders. Besides the elegance of the idea of boosting the body's own defenses, the technology offers another big advantage over traditional drugs: once they have done their job, the engineered T-cells stick around in the body, offering protection against re-infection or recurrence of a cancer possibly for a decade or more. Also, CAR-T could be combined with other therapies to perhaps provide durable cures for certain types of blood cancer and, hopefully, also other kinds of tumors while also better controlling deleterious side effects some of which could be fatal. Thus, in the so-called "triple-attack" treatment, surgery is followed by focused radiation therapy to ablate lesions that may have metastasized, and further followed by a course of chemotherapy. One example of this approach is the case of melanoma lesions that may have metastasized to the brain. Another approach is provided by liver cancer, where a similar triple attack technique combining chemotherapy, thermal ablation, and hyperthermia provides a highly targeted, yet minimally invasive approach.

\section{A historic action making the first gene therapy available}

On August 30, 2017, the United States Food \& Drug Administration (FDA) approved a CAR T-cell therapy for the treatment of certain pediatric and young adult patients (up to 25 years of age) with relapsed or refractory B-cell precursor ALL that does not respond to treatment or has relapsed two or more times.

In announcing its historic action making the first gene therapy available in the United States, the FDA ushered in a new approach to the treatment of cancer and other serious and life-threatening diseases. ${ }^{6}$ FDA Commissioner Scott Gottlieb, MD announced it thus: "We're entering a new frontier in medical innovation with the ability to reprogram a patient's own cells to attack a deadly cancer, New technologies such as gene and cell therapies hold out the potential to transform medicine and create an inflection point in our ability to treat and even cure many intractable illnesses." He also emphasized the "cross-agency approach" involved in this approval with both the Oncology Center for Excellence (OCE) and the Center for Biologics Evaluation and Research (CBER) collaborating. He further underlined that this "more collaborative scientific model for review" will be employed in the future, as the agency is committing to "expediting new and groundbreaking therapies." He lastly noted that there are currently more than 550 investigational new drug applications (INDA) for gene therapy products, including 76 related to CAR T-cells.

The FDA approved the treatment to be trade-named Kymriah (generic name: tisagenlecleucel, also known as CTL019). It granted Kymriah "priority review" and "breakthrough therapy" designations. The Kymriah application was reviewed using a coordinated, crossagency approach. The clinical review was coordinated by OCE while CBER conducted all other aspects of the review and made the final product approval determination.

The FDA approval of Kymriah was granted to Novartis Pharmaceuticals Corp., the drug's manufacturer. It also granted the expanded approval of Actemra to Genentech Inc. The drug will be sold by Novartis at the cost of $\$ 475,000$ for a one-time treatment. A post-marketing study would be required for tisagenlecleucel. This is a crucial part of the new, accelerated pathways to approval.

In addition to a boxed warning and post-marketing study, the FDA is also requiring a Risk Evaluation and Mitigation Strategy (REMS) that includes mandatory training in CRS and its management for all personnel "involved in the prescribing, dispensing, or administering" of tisagenlecleucel. Clinics must also certify that they have tocilizumab on hand for immediate administration.

While the importance of CAR-T cannot be overstated, this approval only pertains to a small population of children. More research is needed to: (a) make this therapy more effective for a broader population, (b) reduce the severe side effects that patients experience during treatment, and, ultimately, (c) find a broader application beyond blood cancers. Continued research will also lead to improved manufacturing of large numbers of cells, which is necessary to make this therapy accessible to more patients.

\section{The Kymriah cell-based gene therapy}

\section{Materials and methods}

Kymriah is a genetically-modified autologous T-cell immunotherapy. Each dose of Kymriah is a customized treatment created using an individual patient's own T-cells.

\section{Approach}

The patient's T-cells are collected and sent to a manufacturing center where they are genetically modified to include a new gene that contains a specific protein (a CAR) that directs the T-cells to target 
and kill leukemia cells that have a specific antigen (CD19) on the surface. Once the cells are modified, they are infused back into the patient to kill the cancer cells. The process takes about 22 days.

\section{Treatment with Kymriah}

Kymriah is approved for use in pediatric and young adult patients with B-cell ALL. It is intended for patients whose cancer has not responded to or has returned after initial treatment, which occurs in an estimated $15-20 \%$ of patients. It is a first-of-its-kind treatment approach that fills an important unmet need for children and young adults with this serious disease. Not only does Kymriah provide these patients with a new treatment option where very limited options existed, but a treatment option that has shown promising remission and survival rates in clinical trials.

\section{Results}

The treatment was initially tested at the University of Pennsylvania in 2010 among adults with advanced chronic lymphocytic leukemia (CLL). In 2012, the Children's Hospital of Philadelphia (CHOP) became the first institution to test the treatment in children with ALL. Since then, the treatment has been evaluated at 13 U.S. medical centers as well as some in other countries. The CHOP trial saw remission at a remarkably improved success rate compared to previous treatment success rates.

Another CAR T-cell therapy, produced by Kite Pharma, is expected to be approved later in 2017 for the treatment of some patients with lymphomas. With the success seen in blood cancers, researchers at NCI and other organizations are busy developing ways to expand the use of CAR T-cell therapies.

\section{Discussion}

Safety and efficacy: The safety and efficacy of Kymriah were demonstrated in one multicenter clinical trial of 63 pediatric and young adult patients with relapsed or refractory B-cell precursor ALL. The overall remission rate within three months of treatment was $83 \%$. However, notwithstanding its high success rate, treatment with Kymriah has the potential to cause severe side effects especially (a) a cytokine release syndrome (CRS) (also dubbed "cytokine storm"), which is a systemic response to the activation and proliferation of CAR T-cells causing high fever and flu-like symptoms that can become lifethreatening, and (b) neurological events. Both CRS and neurological events can be life-threatening. Other severe side effects of Kymriah include serious infections, low blood pressure (hypotension), acute kidney injury, fever, and decreased oxygen (hypoxia). Most symptoms appear within one to 22 days following infusion of Kymriah. Since the CD19 antigen is also present on normal B-cells, Kymriah will also destroy those normal B-cells that produce antibodies, so there may be an increased risk of infections for a prolonged period of time.

Risk of cytokine release syndrome: Some patients in the trial experienced serious side effects associated with the treatment. The infusion of CAR T-cells can lead to inflammatory side effects - the effects known collectively as cytokine release syndrome (CRS)-that range from high fevers and mild flu-like symptoms to life-threatening drops in blood pressure, vascular leakage, and kidney problems. In addition, neurological events, such as confusion and seizures, are also possible and may be life-threatening.

In the trial that led to FDA approval, CRS was managed using the arthritis drug Actemra (tocilizumab), which seems to blunt the symptoms of CRS in the vast majority of patients.
FDA has now expanded the approval of Actemra to treat severe or life-threatening CRS in patients over the age of 2. In clinical trials involving CAR T-cells, $69 \%$ of the patients who received one or two doses of Actemra experienced a complete resolution of CRS within two weeks, according to FDA.

FDA is requiring that institutions offering Kymriah be certified to demonstrate that they have the expertise to treat patients and monitor them for potential side effects. Certification will involve, for instance, training staff to recognize and manage CRS and neurological events.

In an unusual move, the FDA also approved a new indication for the rheumatologic drug Actemra (tocilizumab) to treat CAR T-cellinduced severe or life-threatening CRS in patients 2 years of age or older. In clinical trials in patients treated with CAR-T cells, the Agency stated that " $69 \%$ of patients had complete resolution of CRS within two weeks following one or two doses of Actemra" (which inhibits interleukin-6 action).

Because of the risk of CRS and neurological events, as stated earlier, Kymriah is being approved with REMS, which includes elements to assure safe use (ETASU). The FDA is requiring that hospitals and their associated clinics that dispense Kymriah be specially certified. As part of that certification, staff involved in the prescribing, dispensing, or administering of Kymriah are required to be trained to recognize and manage CRS and neurological events. Additionally, the certified health care settings are required to have protocols in place to ensure that Kymriah is only given to patients after verifying that Actemra is available for immediate administration. The REMS program specifies that patients be informed of the signs and symptoms of CRS and neurological toxicities following infusion - and of the importance of promptly returning to the treatment site if they develop fever or other adverse reactions after receiving treatment with Kymriah.

To further evaluate the long-term safety, the manufacturer Novartis is also required to conduct a post-marketing observational study involving patients treated with Kymriah.

\section{Future prospects}

While the FDA approval is a milestone for the field, it is only the beginning. CAR T-cells that will be tested in different types of cancers. While many of the patients in the trial who experienced remission had run-out of treatment options before starting the CAR T-cell therapy, future CAR T- cell techniques will be tested earlier in the treatment process, rather than when patients have run-out of treatment options.

Follow-up care for patients with ALL who receive CAR T-cells includes monthly outpatient immunoglobulin infusions to prevent infections. Patients have an increased risk of infections because CD19 is present on normal B cells (as well as cancerous ones), and these normal cells are also destroyed by CAR T-cell therapy.

Too many questions must be addressed before immunotherapeutic approaches to cancer could be heralded as an unqualified success. There are still too many severe reactions, too many non-responses or relapses and, potentially, a very high price tag for their widespread use, which will be truly challenging to scale-up.

Among new therapies being developed at NCI, two other forms of adoptive cell transfer are worth noting: T-cell receptor (TCR) and tumor infiltrating lymphocyte (TIL) therapies. NCI researchers are currently conducting many clinical trials testing CAR T-cell, TCR, and TIL therapies in children and adults with cancer. The aim is to 
improve the efficacy of these cellular immunotherapies and finding ways to make them applicable to as many cancer types as possible.

\section{Summary and conclusions}

Beginning with the earlier discoveries of cytotoxic lymphocyte antigen-4 (CTL-4) and programmed death-1 (PD-1), immunotherapy has rapidly evolved during the past decade. With the accumulated knowledge of cancer biology and the immune response system, we have now progressed beyond the stage of merely working with what nature offers us. We are no longer limited to the use of natural cancer biology but have progressed to the use of synthetic biology. That has enabled us to overcome some natural limitations. By genetically modifying the T-cells, we can direct them to home-in directly on antigens that may be abundant on cancer cells.

The technology has however its limitations as we currently know of no other molecule than CD19 that is a specific cancer target. Another limitation is its toxic effects but we may be able in the future to attenuate if not eliminate their effects. The technology can be refined so as not to exclusively depend on the presence of CD19, and can be tailored to patients so as to avoid the deleterious effects of cytokine overproduction (or storms) that could be fatal for some.

Lastly, the technology can be improved by combining it with other complementary therapeutic approaches in a multi-prong attack (a combination of some or all of the following: surgery, radiation therapy, chemotherapy, thermal ablation). The future is very promising, indeed, and we can foresee the CAR T approach being successfully tried in many forms of cancer. ${ }^{7-18}$

\section{Acknowledgements}

None.

\section{Conflicts of interest}

None.

\section{Funding}

None.

\section{References}

1. Fymat AL. Immunotherapy: An Emergent Anti-Cancer Strategy. $J$ Cancer Prev Curr Res. 2017;7(3):1-7.
2. FDA News Release. 2017.

3. Maus MV, Fraietta JA, Levine BL, et al. Adoptive Immunotherapy for Cancer or Viruses. Annu Rev Immunol. 2014;32:189-225.

4. Maude SL, Frey N, Shaw PA, et al. Chimeric Antigen Receptors T-Cells for Sustained Remissions in Leukemia. $N$ Engl J Med. 2014;371(16):1507-1517.

5. Posey AD, June CH, Levine BL. Cancer Killers. Sci Am. 2017;16(3):3843.

6. Federal Register. 2001;6(12).

7. Kruse CA, Merchant RE. Cellular therapy of brain tumors: Clinical trials. Futura Publ Co, USA. 1997; 487-504.

8. Paul DB, Kruse CA. Immunologic approaches to therapy for brain tumors. Curr Neurol Neurosci Rep. 2001;1(3):238-244

9. Yang MY, Zetler PM, Prins RM, et al. Immunotherapy for patients with malignant glioma: From theoretical principles to clinical applications. Expert Rev Neurother. 2006;6(10):1481-1494.

10. u X, Stockhammer F, Schmitt M. Cellular-based immunotherapies for patients with glioblastoma multiform. Clinical and Developmental Immunology. 2012:15.

11. Reardon DA, Wucherpfennig KW, Freeman G, et al. An update on vaccine therapy and other immunotherapeutic approaches for glioblastoma. Expert Rev Vaccines. 2013;12(6):597-615.

12. Fymat AL. Synthetic Immunotherapy with Chimeric Antigen Receptors. J Cancer Prev Curr Res. 2017;7(5):00253.

13. Maude SL. Chimeric Antigen Receptors T-Cells for Sustained Remissions in Leukemia. N Engl J Med. 2014;371(16):1507-1517.

14. Maus MV, Fraietta JA, Levine BL, et al. Adoptive Immunotherapy for Cancer or Viruses. Annual Review of Immunology. 2014;32:189-225.

15. Posey AD, June CH, Levine BL. Cancer Killers. Sci Am. 2017;16(3):3843.

16. Serres S, Anthony DC, Jiang Y, et al. Systemic inflammatory response reactivates immune-mediated lesions in rat Bbain. $J$ Neurosci. 2009;29(15):4820-4828

17. Valera E, Masliah E. Immunotherapy for neurodegenerative diseases: Focus on $\alpha$-synucleinopathies. Pharmacology Therap. $2013 ; 138(3): 311-322$

18. Hutter-Saunders JA, Mosley RL, Gendelman HE. Pathways towards an effective immunotherapy for Parkinson's disease. Expert Rev Neurother. 2011;11(12):1703-1715. 


\section{Appendix A}

\section{What is Human Gene Therapy?}

\section{Definition}

Human gene therapy (HGT) is the administration of genetic material into a person's DNA to replace faulty or missing genetic material, i.e. to modify or manipulate the expression of a gene product or to alter the biological properties of living cells for the purpose of treating a disease or abnormal medical condition.

\section{Regulatory Agency}

The (U.S.) FDA's Center for Biologics Evaluation and Research (CBER) is the Federal Government's Agency approving the use of biologic products such as gene therapy products. It also regulates certain devices related to gene therapy. CBER uses both the Public Health Service Act (PSHA) and the Federal Food Drug and Cosmetic Act (FDCA) as enabling statutes for oversight.

\section{Mechanisms}

Gene therapies can work by several mechanisms:

i. Replacing a disease-causing gene with a healthy copy of the gene;

ii. Inactivating a disease-causing gene that is not functioning properly; or else

iii. Introducing a new or modified gene into the body to help treat a disease.

\section{Products}

Gene therapy products are biological products regulated by the FDA's Center for Biologics Evaluation and Research (CBER). In the United States, clinical studies in humans require the submission of an investigational new drug application (INDA) prior to initiating clinical studies. Marketing a gene therapy product requires submission and approval of a biologics license application (BLA) [6].

Gene therapy products are being studied to treat diseases including cancer, genetic diseases, and infectious diseases. There are various gene therapy products, including:

a) Plasmid DNA: Circular DNA molecules that can be genetically engineered to carry therapeutic genes into human cells;

b) Viral vectors: Viruses have a natural ability to deliver genetic material into cells and, therefore, some gene therapy products are derived from viruses [1,2]. Once viruses have been modified to remove their ability to cause infectious disease, these modified viruses can be used as vectors (vehicles) to carry therapeutic genes into human cells; c) Bacterial vectors: Bacteria can be modified to prevent them from causing infectious diseases and then used as vectors to carry therapeutic genes into human tissues;

d) Human gene editing technology: The goals of gene editing are to disrupt harmful genes or to repair mutated genes; and

e) Patient-derived cellular gene therapy products: Cells are removed from the patient, genetically modified (often using a viral vector) and then returned to the patient.

\section{Research and Development Growth}

Gene therapy-related research and development in the United States continues to grow at a fast pace with a number of products advancing to clinical development. In addition to regulatory oversight of clinical studies, the FDA's Center for Biologics Evaluation and Research (CBER) provides proactive scientific and regulatory advice to medical researchers and manufacturers in the area of novel product development.

\section{Appendix B}

\section{Regulatory agency}

The FDA's Center for Biologics Evaluation and Research (CBER) is the Agency regulating the use of cellular therapy products. It also regulates certain devices. CBER also regulates certain devices related to cell therapy. It uses both the Public Health Service Act (PSHA) and the Federal Food Drug and Cosmetic Act (FFDCA) as enabling statutes for oversight.

\section{Products}

i. Cellular therapy products include:

ii. Cellular immunotherapies;

iii. Cancer vaccines; and

iv. Other types of both autologous and allogenic cells for certain therapeutic indications, including :

v. Hematopoietic stem cells, and

vi. Adult and embryonic stem cells.

\section{Research \& Development Growth}

Cellular research and development in the United States continues to grow at a fast pace, with a number of products advancing in clinical development. In addition to regulatory oversight of clinical studies, CBER provides proactive scientific and regulatory advice to medical researchers and manufacturers in the area of novel product development. 